\section{A Y2H-seq approach defines the human protein methyltransferase interactome}

\author{
Mareike Weimann ${ }^{1,4}$, Arndt Grossmann ${ }^{1,4}$, \\ Jonathan Woodsmith ${ }^{1,4}$, Ziya Özkan ${ }^{1}$, Petra Birth ${ }^{1}$, \\ David Meierhofer ${ }^{1}$, Nouhad Benlasfer ${ }^{1}$, \\ Taras Valovka ${ }^{2}$, Bernd Timmermann ${ }^{1}$, \\ Erich E Wanker ${ }^{3}$, Sascha Sauer ${ }^{1}$ \& Ulrich Stelzl ${ }^{1}$
}

\begin{abstract}
To accelerate high-density interactome mapping, we developed a yeast two-hybrid interaction screening approach involving short-read second-generation sequencing ( $\mathrm{Y} 2 \mathrm{H}-\mathrm{seq}$ ) with improved sensitivity and a quantitative scoring readout allowing rapid interaction validation. We applied $\mathrm{Y} 2 \mathrm{H}$-seq to investigate enzymes involved in protein methylation, a largely unexplored post-translational modification. The reported network of 523 interactions involving 22 methyltransferases or demethylases is comprehensively annotated and validated through coimmunoprecipitation experiments and defines previously undiscovered cellular roles of nonhistone protein methylation.
\end{abstract}

Despite recent conceptual and technical advances ${ }^{1-3}$, large-scale interactome mapping remains a daunting task, and for most species, only a small fraction of interactions have been mapped. Yeast two-hybrid $(\mathrm{Y} 2 \mathrm{H})$ analysis can be applied at high throughput and contributes substantially to high-quality interactome mapping ${ }^{3-5}$. Empirical assessment of data quality has demonstrated that $\mathrm{Y} 2 \mathrm{H}$ protein-protein interaction (PPI) data are of high precision but of low coverage, owing to low sensitivity of the methods ${ }^{1}$, and therefore require repeated screens to capture most detectable interactions. We used an Illumina sequencing readout to modify the $\mathrm{Y} 2 \mathrm{H}$ screening procedure ( $\mathrm{Y} 2 \mathrm{H}$-seq), which led to higher sampling of interacting protein pairs, an increase in sensitivity and a quantitative score of interaction probability.

We applied $\mathrm{Y} 2 \mathrm{H}$-seq for interactome mapping of a largely unexplored set of human proteins: those involved in protein methylation and demethylation ${ }^{6}$. From comparison with other abundant modifications, it has become clear that protein methylation of arginine $(\mathrm{R})$ and lysine $(\mathrm{K})$ residues is an important, ubiquitous post-translational modification in eukaryotic cells ${ }^{6,7}$.
A 'writer-reader-eraser' post-translational modification regulatory system consisting of a large number of methyltransferases ${ }^{8,9}$, methyl-recognition domain-containing proteins ${ }^{10}$ and putative demethylases ${ }^{11}$ are expressed in different subcellular locations in humans, an indication that protein methylation is involved in processes other than epigenetic regulation.

We prepared $82 \mathrm{Y} 2 \mathrm{H}$ bait strains spanning human R-methyltransferases (PRMT1-PRMT8) ${ }^{8}, 16$ SET domaincontaining K-methyltransferases (PKMTs) ${ }^{9}$, 9 members of the JMJD domain-containing protein family of protein demethylases and AOF2 (LSD1) ${ }^{11}$ (Supplementary Table 1). In our current matrix screening protocol ${ }^{4,12}$, we perform four replicates, testing every set of baits individually against each of the $\sim 13,000$ prey contained in the matrix. Interacting prey are identified by their position in the matrix. To increase the sensitivity of the approach while also reducing the workload, we used a pooled strategy to test each protein pair substantially more than four times. Baits were pooled with all prey strains and then assayed for interaction in more than 100,000 separate spots (Fig. 1a and Supplementary Fig. 1). Using $\mathrm{Y} 2 \mathrm{H}$-seq, we obtained 4-10 times the number of positive colonies obtained with the matrix approach. To reveal the prey identities, we collected all colonies and performed a 36-base parallel sequencing run. More than 20 million reads mapped perfectly to human RefSeq coding sequences (open reading frames, ORFs), corresponding to more than 500,000 unique 36-base reads (Supplementary Table 2). To rank the potentially interacting proteins for subsequent interaction retesting, we calculated a 'SeqScore' that incorporates the number of total mappings and the number of unique reads matching the ORF (Supplementary Fig. 2). Notably, $>99.7 \%$ of the RefSeq mappings matched to the 400 top-ranked genes, thus allowing the identification of potentially interacting ORFs with an extremely high signal-tobackground ratio (Supplementary Table 2).

We performed four biological replicates and demonstrated in statistical pairwise comparisons that they result in very similar ranked prey orders (Supplementary Fig. 3). Top-ranked prey in at least two replicate screens were retested against all baits in a pairwise manner (Supplementary Fig. 4) and yielded 463 protein interactions (Supplementary Table 3). The success rate of the retest-that is, the probability that the prey is interactingdecreased with decreasing SeqScore (Fig. 1b).

We also performed a matrix screen in quadruplicate with a subset of the protein methyltransferase (PMT) and protein demethylase (PDeM) baits for direct method comparison. With the matrix approach, we found 151 interactions (Supplementary Table 3) with 90 prey proteins at a retest success rate of 0.78 . Comparison of the $\mathrm{Y} 2 \mathrm{H}$-matrix and the $\mathrm{Y} 2 \mathrm{H}$-seq approaches at the same

${ }^{1}$ Otto-Warburg Laboratory, Max Planck Institute for Molecular Genetics (MPIMG), Berlin, Germany. ${ }^{2}$ Institute of Biochemistry, Center for Molecular Biosciences, University of Innsbruck, Innsbruck, Austria. ${ }^{3}$ Neuroproteomics Program, Max Delbrück Center for Molecular Medicine, Berlin-Buch, Germany. ${ }^{4}$ These authors contributed equally to this work. Correspondence should be addressed to U.S. (stelzl@molgen.mpg.de). 
a

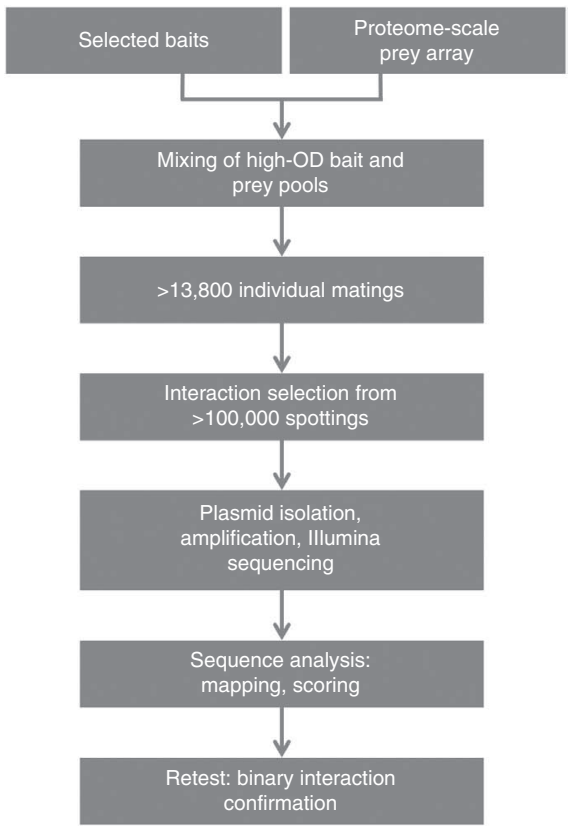

b
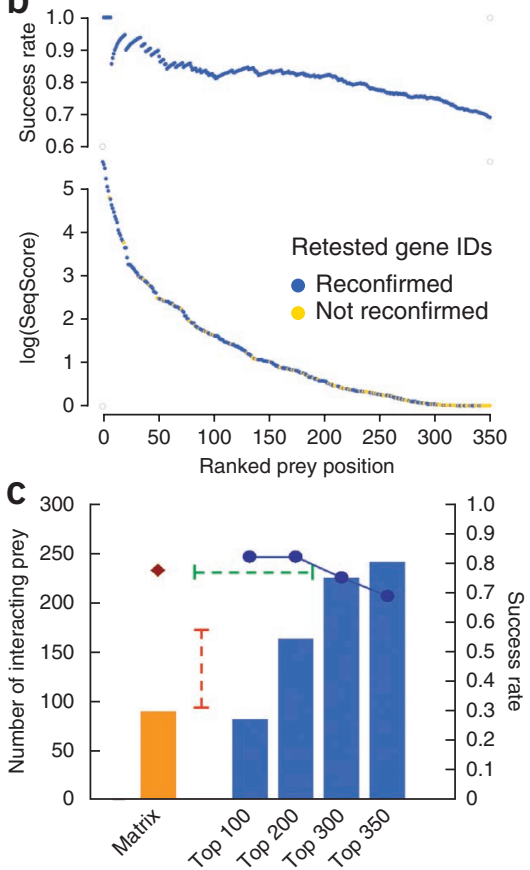

Figure 1 | The $\mathrm{Y} 2 \mathrm{H}$-seq approach. (a) Schematic describing the major steps in $\mathrm{Y} 2 \mathrm{H}$-seq. Baits (82) and prey $(\sim 13,000)$ were grown individually in array format and then pooled. The mixture was cultured in a grid pattern on nonselective agar to allow several thousand separate pooled matings in distinct colonies, each potentially containing all bait-prey combinations. The colonies were transferred to selective medium to identify interacting bait-prey pairs. Colonies were collected, and prey DNA was isolated and sequenced (one lane on Illumina Genome Analyzer). Scored prey were subjected to a pairwise retest. (b) Cumulative retest success rate (top) and the SeqScore distribution (bottom) as a function of prey rank position. (c) Number of interacting prey from the matrix approach (orange bar) and from the top 100, 200, 300 and 350 retested $\mathrm{Y} 2 \mathrm{H}$-seq candidate proteins (blue bars). Retest success rates for the matrix (diamond) and the $\mathrm{Y} 2 \mathrm{H}$-seq (circles) approaches are also shown. $\mathrm{Y} 2 \mathrm{H}$-seq yielded about twice as many interacting prey (red dashed line) as the matrix approach at the same retest success rate (green dashed line). retest success rate demonstrates that the sensitivity of the $\mathrm{Y} 2 \mathrm{H}$ seq approach was approximately double (Fig. 1c). As the $\mathrm{Y} 2 \mathrm{H}$ system and retest procedure were identical in both approaches, we conclude that, at the same specificity, $\mathrm{Y} 2 \mathrm{H}$-seq has a substantially improved sampling sensitivity.
Two previous studies replaced the Sanger sequencing readout of individual $\mathrm{Y} 2 \mathrm{H}$ pairs with second-generation sequencing 33,13 , which also reduced workload and cost but did not lead to an increase in sensitivity in their current assay implementations. One of these studies ${ }^{13}$ involved a cDNA library screen and identified
Figure 2 | The protein methyltransferase interactome. (a) Pairwise overlap of interaction partners of protein methyltransferase (PMTs) and protein demethylase (PDeMs). Yellow to red (0 to 1): number of common prey between the indicated proteins divided by number of total interaction partners of the protein in the row (indicated to the right). The bottom row shows the fraction of prey identified with only the indicated protein. Asterisk indicates statistically significant overlap $(P=0.002)$.

(b) Enrichment analysis for protein functions (left) and protein domains (right) in interacting proteins. The fractions of enriched proteins $(P<0.05)$ among the interaction partners of the indicated PTM or PDeM (color coded) are shown. The categories were also enriched in the whole data set $\left(1.2 \times 10^{-9}<P<0.03\right)$. Asterisks indicate enrichment of RNA bindingand WD40-repeat domain-containing proteins in the set of JMJD6 and PRMT1 interaction partners, respectively. These two categories were not enriched in the whole data set. act., activity; bdg., binding. (c) Cellular component annotation of interacting proteins. Outer ring, Gene Ontology (G0) cellular component annotation for 278 of 324 interacting proteins. Proteins with both G0:0005634 (nucleus) and G0:0005737 (cytoplasm) annotation were grouped in the "cytoplasm + nucleus" category. Nucleolar proteins are not contained in the nucleus annotation, and proteins in the cytosol are not assigned to the cytoplasm if they have both annotations. Inner ring, nonredundant annotation of interacting proteins in four major component categories: nucleus, cytoplasm + nucleus, cytoplasm and other.
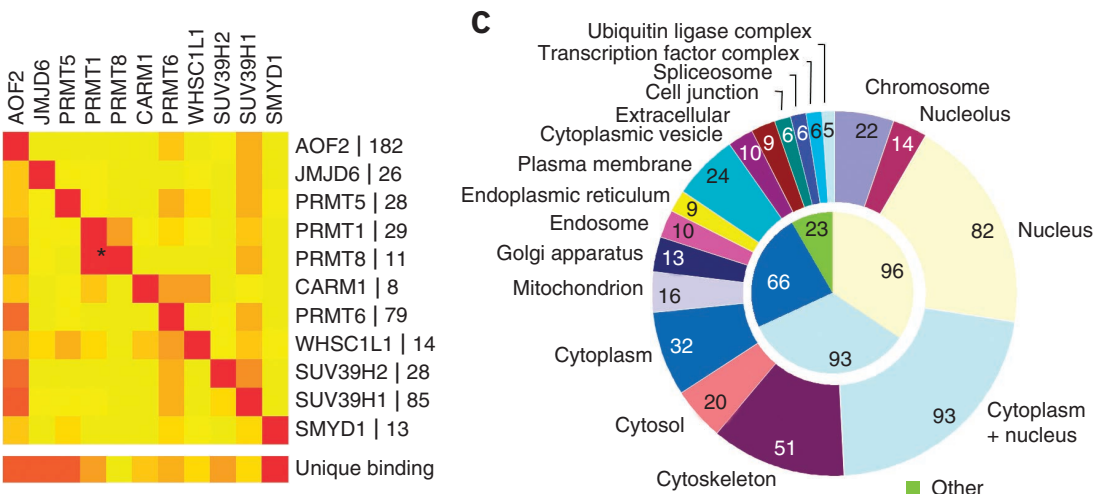

b
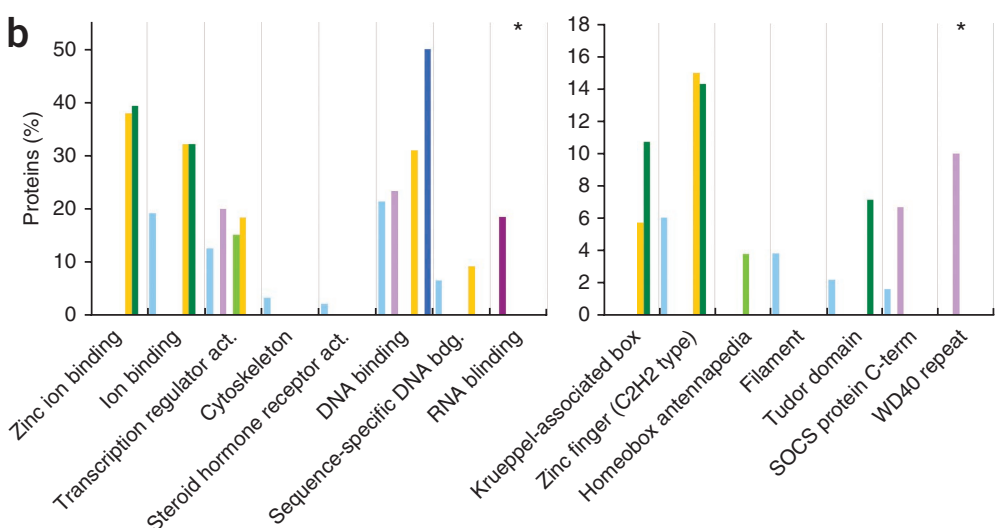
Figure 3 | Validation of the $\mathrm{Y} 2 \mathrm{H}$-seq data. (a) $\log _{2}$-scaled luciferase activity for 20 candidate PRMT1-interacting proteins compared to activity of noninteracting control (ctrl) proteins after co-IP (Online Methods). Dots indicate recapitulated interactions with greater than twofold binding over control and $z$ score $>2$. Error bars, s.d. $(n=3)$. For the full co-IP data set (179 PPI pairs), see Supplementary Tables 4 and 5. (b) Methylation (Me) sites of purified interacting proteins identified through in vitro methylation followed by LC-MS/MS peptide scanning. All listed sites were found using HEK293 cell lysate; sites labeled with an asterisk were also found with $E$. coli lysate that contained CARM1 or PRMT1, respectively. R491 of SYNCRIP and R493 of PABPC1 are known methylation sites.

11 HopZ2 candidate interacting proteins via Illumina sequencing. The other study ${ }^{3}$ involved a two-step PCR protocol linking bait and prey for en masse sequencing with 454 FLX technology (Stitch-seq). Although Stitch-seq enables testing of a large interaction space, assay sensitivity is not apparently increased, and the resulting data are relatively sparse (979 interactions from systematic screening of 36 million protein pairs ${ }^{3}$ ). We note, however, that improved sequencing technology or alternate workflows may increase the usable read count and may allow deeper sequencing of the stitch-PCR products in the future. Crucially, interactome mapping requires both search space and interaction coverage: that is, scalability in the number of protein pairs that can be assayed and high sensitivity to actually detect the interactions in the search space. More than 20 million reads map to the proteins identified in our Y2H-seq approach (Supplementary Table 2), which indicates good scalability for sequencing hundreds of separate screens through barcode indexing in a single Illumina run. Its increased sensitivity leads to higher interaction coverage in the screened interactome space.

We report 523 unique interactions involving 22 PMTs and PDeMs and 324 interacting proteins (Supplementary Fig. 5 and Supplementary Table 3). The largest numbers of interactions were found with the lysine demethylase AOF2 (182), and more than 25 interactions each were identified for SUV39H1, PRMT6, PRMT1, JMJD6, SUV39H2 and PRMT5. Aside from the most closely related R-methyltransferases, PRMT1 and PRMT8 (ref. 8), the majority of enzymes show distinct interaction partner preferences (Fig. 2a and Supplementary Figs. 4 and 5), consistent with notable nonoverlapping roles for each PMT and PDeM.

Functional enrichment analyses suggested specific cellular functions from the binding profiles of distinct enzyme subgroups (Fig. 2b). For example, SUV39H1 and SUV39H2 showed enriched binding to zinc-finger ( $\mathrm{C} 2 \mathrm{H} 2$ type)-containing proteins, and homeobox domain-containing transcription factors were associated with PRMT6. Furthermore, AOF2-interacting proteins were enriched in filament domains because these proteins include a set of cytoskeletal proteins and keratins (Fig. 2b and Supplementary Fig. 5). In agreement with targeted studies, interacting partners of PMTs and PDeMs were prominent in transcriptional regulation. PMTs and PDeMs may be recruited to function as co-regulators in transcription modulating the modifications of histones, nuclear receptors, other co-regulators or signal-transduction components ${ }^{7}$. The PPI data also pointed toward new roles for protein methylation, with more than half of the proteins identified residing at least partially outside of the nucleus (Fig. 2c).

To experimentally validate the $\mathrm{Y} 2 \mathrm{H}$ interactions in an independent assay, we selected a large fraction of interactions

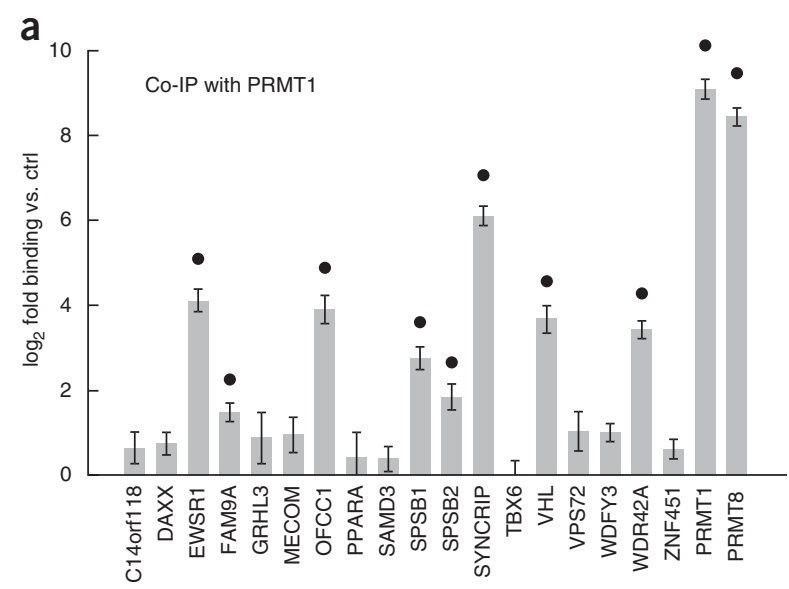

\begin{tabular}{|c|c|c|}
\hline Symbol & Protein name & Me site \\
\hline QKI & Protein quaking & $\mathrm{R} 242$ * \\
\hline DNAJA3 & DnaJ homolog & $\begin{array}{l}\mathrm{R} 58^{*} \\
\mathrm{R} 238^{*} \\
\mathrm{R} 293^{*}\end{array}$ \\
\hline PABPC1 & $\begin{array}{l}\text { Poly(A)-binding protein } 1 \\
\text { (PABP1) }\end{array}$ & $\begin{array}{l}\mathrm{R} 419 \\
\mathrm{R} 436 \text { * } \\
\mathrm{R} 493 \text { * }\end{array}$ \\
\hline OFCC1 & $\begin{array}{l}\text { Orofacial cleft } 1 \text { candidate } \\
\text { gene } 1 \text { protein }\end{array}$ & $\mathrm{R} 113$ \\
\hline WDR42A & $\begin{array}{l}\text { DDB1- and CUL4-associated } \\
\text { factor } 8 \text { (DECAF8) }\end{array}$ & $\mathrm{R} 204$ * \\
\hline SYNCRIP & $\begin{array}{l}\text { Heterogeneous nuclear } \\
\text { ribonucleoprotein Q } \\
\text { (hnRNP-Q) }\end{array}$ & $\begin{array}{l}\mathrm{R} 409 \\
\mathrm{R} 461 \\
\mathrm{R} 475 \\
\mathrm{R} 483 \\
\mathrm{R} 491 \text { * } \\
\mathrm{R} 498 \\
\mathrm{R} 504 \\
\mathrm{R} 507\end{array}$ \\
\hline
\end{tabular}

involving our major interacting PMTs and PDeMs and performed immunofluorescence-based coimmunoprecipitation (co-IP) assays with human cells. Overall, the co-IP assays recapitulated $\sim 64 \%$ of the 179 interactions tested (Supplementary Tables 4 and 5), underscoring the high quality of our PPI data set (see Supplementary Fig. 6 for benchmarking against the literature). Interacting proteins coprecipitated AOF2 most efficiently (78\%), and 10 of 20 tested PRMT1 interactions were validated under the conditions used (Fig. 3a), which suggests that a large fraction of PRMT1 interaction partners may interact rather transiently. In addition to reconfirming three of its known partners ${ }^{14}$, we recapitulated newly identified PRMT1-interacting partners in the co-IP experiments (Fig. 3a), such as the SOCS proteins SPSB1 and SPSB2, the von Hippel-Lindau VHL protein and WDR42A (DCAF8), all of which are adaptor proteins for different CullinRING ubiquitin ligases ${ }^{15}$.

PMT- and PDeM-interacting proteins may regulate enzyme activity or act as recruitment factors or as methylation substrates. Only a relatively small number of proteins are known to be methylated on arginine and lysine residues, some of which are found among our interacting proteins (Supplementary Table 6). To test whether proteins can be methylated in vitro, 
we analyzed seven candidate proteins treated with HEK293 or Escherichia coli lysates containing CARM1 or PRMT1 using liquid chromatography-tandem mass spectrometry (LC-MS/MS) scanning (Fig. 3b and Supplementary Table 7). We identified known and novel arginine methylation sites on SYNCRIP and PABPC1, and we detected methylation of the PRMT1-interacting proteins WDR42A and OFCC1 and the CARM1-interacting proteins DNAJA3 and QKI (quaking). QKI was previously reported to be methylated, but neither the site nor the responsible enzyme was identified ${ }^{16}$. We provide evidence that R242 can be methylated by CARM1 and suggest that this could alter QKI function in regulated mRNA export ${ }^{17}$. These experiments should be viewed as proof of concept that identified PRMT1- and CARM1-interacting proteins can be methylated on arginine residues. Together with the PPI data, these data suggest that whether these cases are in vivo substrate-enzyme pairs be further investigated.

We have applied $\mathrm{Y} 2 \mathrm{H}$-seq to generate a large, comprehensively annotated resource describing a partially validated set of interaction partners for proteins involved in methylation, providing hints toward novel cellular functions for arginine and lysine protein methylation.

\section{METHODS}

Methods and any associated references are available in the online version of the paper.

Accession codes. The interaction data are accessible via MINT, the molecular interaction database, under the IMEx identifier IM-17917.

Note: Supplementary information is available in the online version of the paper.

\section{ACKNOWLEDGMENTS}

We thank B. Lukaszewska-McGreal from the MPIMG-mass spectrometry group and D. Roth and S. Paturej from the MPIMG-next-generation sequencing team for their experimental support. The work was supported by the Max Planck Society and the German Ministry of Science (NGFNp, NeuroNet-TP3 01GS08171; BMBF, 0315082).

\section{AUTHOR CONTRIBUTIONS}

M.W. performed $\mathrm{Y} 2 \mathrm{H}$ screens. A.G. and U.S. developed the screening protocol. J.W. and A.G. analyzed the sequencing data. Z.0̈. contributed to cloning, $\mathrm{Y} 2 \mathrm{H}$ and protein purification experiments. P.B. performed the co-IP experiments. M.W. and N.B. performed the methylation assays. D.M. performed the mass spectrometry analysis. T.V., B.T., E.E.W. and S.S. contributed tools and reagents. M.W., J.W. and U.S. analyzed the data and generated the figures. U.S. conceived and supervised the study and wrote the manuscript. All authors provided feedback on the manuscript.

\section{COMPETING FINANCIAL INTERESTS}

The authors declare no competing financial interests.

Reprints and permissions information is available online at http://www.nature. com/reprints/index.html.

1. Venkatesan, K. et al. Nat. Methods 6, 83-90 (2009).

2. Schwartz, A.S., Yu, J., Gardenour, K.R., Finley, R.L. Jr. \& Ideker, T. Nat. Methods 6, 55-61 (2009)

3. Yu, H. et al. Nat. Methods 8, 478-480 (2011).

4. Vinayagam, A. et al. Sci. Signal. 4, rs8 (2011).

5. Hegele, A. et al. Mol. Cell 45, 567-580 (2012).

6. Erce, M.A., Pang, C.N., Hart-Smith, G. \& Wilkins, M.R. Proteomics $\mathbf{1 2}$ 564-586 (2012).

7. Lee, Y.H. \& Stallcup, M.R. Mol. Endocrinol. 23, 425-433 (2009).

8. Bedford, M.T. \& Clarke, S.G. Mol. Cell 33, 1-13 (2009).

9. Huang, J. \& Berger, S.L. Curr. Opin. Genet. Dev. 18, 152-158 (2008).

10. Chen, C., Nott, T.J., Jin, J. \& Pawson, T. Nat. Rev. Mol. Cell Biol. 12, 629-642 (2011).

11. Lan, F., Nottke, A.C. \& Shi, Y. Curr. Opin. Cell Biol. 20, 316-325 (2008).

12. Worseck, J.M., Grossmann, A., Weimann, M., Hegele, A. \& Stelzl, U. Methods Mol. Biol. 812, 63-87 (2012).

13. Lewis, J.D. et al. BMC Genomics 13, 8 (2012).

14. Passos, D.0., Bressan, G.C., Nery, F.C. \& Kobarg, J. FEBS J. 273 , 3946-3961 (2006).

15. Yang, Y. \& Bedford, M.T. Mol. Cell 48, 487-488 (2012).

16. Côté, J., Boisvert, F.M., Boulanger, M.C., Bedford, M.T. \& Richard, S. Mol. Biol. Cell 14, 274-287 (2003).

17. Zhang, Y. et al. EMBO J. 22, 1801-1810 (2003). 


\section{ONLINE METHODS}

Y2H interaction mating. For Y2H analysis, bait pBTM116-D9 and pBTMcC24-DM (identical to pBTM116-D9 except that the lexA DNA-binding protein is fused at the $\mathrm{C}$ terminus of the ORF) and prey pACT4-DM gateway compatible vectors ${ }^{12}$ were used in combination with $\mathrm{L} 40 \mathrm{ccU}$ MATa and $\mathrm{L} 40 \mathrm{cc} \alpha$ MAT $\alpha$ yeast strains $^{18}$, respectively. The matrix-format Y2H PPI screening was performed as described in detail recently ${ }^{12}$. In short, 32 non-autoactivating bait strains were pooled and mated with the arrayed collection of individual prey strains in 384-well format four times. Positive clones were identified by growth on selective medium (-Leu -Trp -Ura -His). Prey strains that were identified two or more times in the pooled mating screens were tested for interactions against individual baits in the retest using fresh yeast cells.

For the $\mathrm{Y} 2 \mathrm{H}$-seq approach, autoactivating strains were removed from the prey array and the set of bait strains. The prey array and the selected bait strains were replicated (in 384- and 96-well format, respectively). The freshly grown yeast colonies were scratched off the agar and resuspended in liquid medium, and the pools of bait and prey strains were mixed in a 1:1 ratio at $\mathrm{OD}_{600}$ of $\sim 70$. The mixed culture $(\sim 10 \mathrm{ml})$ was distributed into a 384-well MTP and transferred in a grid pattern ('gridded') repeatedly on YPD agar. Mating took place in 13,824 distinct spots at $30{ }^{\circ} \mathrm{C}$ for $40 \mathrm{~h}$. Yeast colonies were resuspended in 36 384-well microtiter plates containing -Leu -Trp liquid medium and gridded in high-density $9 \times 384$ format on - Leu -Trp -Ura -His agar plates for interaction selection $\left(124,416\right.$ spots). The plates were incubated at $30^{\circ} \mathrm{C}$ for 5-7 $\mathrm{d}$ to allow growth on selective medium indicating interaction between at least one pair of bait and prey. All yeast colonies grown on -Leu -Trp -Ura -His agar were scratched off, and plasmid DNA was prepared from the yeast pellet using a standard alkaline lysis protocol and ethanol precipitation. The DNA was further purified by phenol extraction, dialyzed against TE buffer and collected through ethanol precipitation. The prey ORF inserts were PCR-amplified with 5'-Hex-blocked primer (the $3^{\prime}$ nucleoside was attached via a phosphothioate) that targeted unique sites $5^{\prime}$ and $3^{\prime}$ to the ORF insert in the prey plasmid. The PCR yielded a DNA smear of the expected size range ( 800 to $>4,000 \mathrm{bp}$ ) for the prey ORFs when applied to agarose gel electrophoresis. DNA (3 $\mu \mathrm{g}$ ) was fragmented and used for single-read, 36-base Illumina sequencing (Illumina Genome Analyzer).

Next-generation sequence analysis. For each replicate, highquality (HQ) 36-base sequences were extracted from the raw sequence data (sequences containing no undetermined base calls). This resulted in $\sim 34-49$ million reads per sequencing run distributed over $\sim 6-9$ million unique reads, highlighting that each unique 36-base read can be represented multiple times. These sequences were then mapped against a pseudogenome composed of the human protein coding sequences from RefSeq (NCBI release 2011-02-16), the Saccharomyces cerevisiae genome and the $\mathrm{Y} 2 \mathrm{H}$ vector sequences. To map the HQ sequences against the pseudogenome, the SHRiMP software package, version 1.2.1, was used with default settings ${ }^{19}$. This resulted in $\sim 20-28$ million reads perfectly mapped to protein coding RefSeq sequences, representing $56 \%-59 \%$ of the total reads mapped in each replicate workflow (Supplementary Table 2). Only reads with perfect alignment to target sequences were taken forward for further analysis.
To generate a ranked retest list of $\mathrm{Y} 2 \mathrm{H}$ prey clones that accurately reflects the mappings, a sequence score was calculated for every RefSeq identifier. To bias against RefSeq identifiers that were mapped with a high number of mappings but with a low number of unique reads, a sequence score (SeqScore) combining the number of mappings and the number of unique reads for each RefSeq protein coding sequence (in both forward and reverse directions) was used.

$$
\text { SeqScore }=\frac{(c d s-35) \times 2}{\sum_{k=1}^{n} \frac{1}{x_{k}}+((c d s-35) \times 2-n)}
$$

cds is the protein-coding sequence length (in bp); $x$ is the number of mappings associated with each unique read $+1 ; n$ is the number of unique reads matching a given RefSeq coding sequence. The SeqScore gives a relatively high value for RefSeq identifiers that were mapped with multiple unique sequences and with high frequency as potential interacting prey for retesting (Supplementary Fig. 2). Entrez Gene ID-based ranked retest lists were generated by taking the maximum SeqScore from the set of associated RefSeq identifiers for each analytical workflow. For the final retest list, we took the second-highest score for each gene ID from the four ranked lists. We therefore excluded gene IDs associated with a high ranking score from only one biological replicate, favoring those that scored highly over at least two. The final, pairwise retest was performed using fresh yeast strains to confirm the interaction of the prey and determine the identity of the interacting bait ${ }^{12,20}$. It was identical in the matrix and $\mathrm{Y} 2 \mathrm{H}$-seq approach. Therefore, specificity of data was the same in both approaches. Previously, we have empirically assessed the quality of large-scale $\mathrm{Y} 2 \mathrm{H}$ data and demonstrated that single-repeat proteome-scale screening data with our $\mathrm{Y} 2 \mathrm{H}$ system have a precision of $\sim 80 \%$ when tested with an independent PPI assay ${ }^{1}$.

Enrichment and annotation analyses. Enrichment analysis was performed with DAVID ${ }^{21}$ using indicated sets of prey (Entrez Gene ID level) with the $\mathrm{Y} 2 \mathrm{H}$ matrix genes as background. The tool reports the modified Fisher exact $P$ value for enrichment and the absolute number of genes for each annotation cluster. Gene Ontology cellular component annotation was performed using Entrez gene2go (12-04-04).

Coimmunoprecipitation analysis. PMT and PDeM ORFs were transferred to firefly-V5 fusion vectors (pcDNA3.1V5-Fire) and interacting proteins to protein A fusion vectors (pcDNA3.1PAD57), respectively ${ }^{5,22}$, using standard Gateway cloning procedures. For co-IP assays, $3 \times 10^{4} \mathrm{HEK} 293$ cells were transiently cotransfected with firefly (75 ng) and protein A (PA; $50 \mathrm{ng}$ ) plasmid DNA using Lipofectamine 2000 (Invitrogen) in a well of a 96-well plate. Cells were lysed $24-30 \mathrm{~h}$ after transfection in $100 \mu \mathrm{l}$ SDS-free RIPA-buffer (50 mM Tris- $\mathrm{HCl}, \mathrm{pH}$ 7.4, $150 \mathrm{mM} \mathrm{NaCl}$, $1 \%$ Nonidet P-40, $0.25 \%$ sodium deoxycholate, $1 \mathrm{mM}$ EGTA and protease inhibitor (Roche, 11051600)) for $30 \mathrm{~min}$ at $4{ }^{\circ} \mathrm{C}$. Protein complexes were precipitated from $70 \mu \mathrm{l}$ cleared cell extract in IgGcoated microtiter plates for $1 \mathrm{~h}$ at $4{ }^{\circ} \mathrm{C}$ and washed three times with $100 \mu \mathrm{l}$ ice-cold PBS. The binding of the firefly-V5-tagged fusion protein (co-IP) to the PA-tagged fusion protein (IP) was 
assessed by measuring the firefly luciferase activity in a luminescence plate reader (Beckmann D TX800, Bright-Glo Luciferase Assay (Promega)). Assays were performed as triplicate transfections. $\log _{2}$-fold binding for a protein pair was calculated from relative luciferase intensities (RLU) in comparison to background binding measured in parallel with the firefly (Fire)- and nonrelated protein A-tagged fusion protein (Ctrl).

$\log _{2}$-fold binding compared to control protein binding was calculated as

$$
\begin{aligned}
\log _{2} \text { fold_binding }= & \operatorname{average}\left(\log _{2}\left(\mathrm{RLU}_{(\text {Fire,PA })}\right)\right) \\
& -\operatorname{average}\left(\log _{2}\left(\mathrm{RLU}_{(\text {Fire }, \text { Ctrl })}\right)\right)
\end{aligned}
$$

Corrected s.d. of an experiment performed as triplicate transfections was calculated as

$$
\begin{aligned}
\log _{2} \text { fold_std_corr }= & \operatorname{StDev}\left(\log _{2}(\mathrm{RLU}(\text { Fire }, \mathrm{PA}))\right) \\
& +\operatorname{StDev}\left(\log _{2}\left(\mathrm{RLU}_{(\text {Fire }, \text { Ctrl })}\right)\right)
\end{aligned}
$$

The $z$-score measure of significance of the difference between the binding of the firefly protein to PA versus to the control was calculated as

$$
z=\log _{2} \text { fold_binding } / \log _{2} \text { fold_std_corr }
$$

Experiments with $\log _{2}$ fold_binding $>1$ (i.e., twofold binding) and a $z$ score $>2$ were considered positive.

In vitro methylation. Using the PRMT1 substrate $\mathrm{SYNCRIP}^{23}$ and the CARM1 substrate PABP1 (ref. 24) for control, we performed in vitro methylation assays. Candidate proteins were expressed as either His6 or GST fusions in E. coli SCS1, and the soluble protein fraction was bound to Ni-NTA or glutathione agarose beads, respectively. The immobilized proteins were treated either with freshly prepared, cleared cell lysate from HEK293 cells or with cleared E. coli lysate overproducing the human PRMT ${ }^{25}$. After $2 \mathrm{~h}$ incubation at $30^{\circ} \mathrm{C}$ in the presence of $100 \mu \mathrm{M}$ AdoMet ( $S$-adenosylmethionine), the immobilized proteins were washed and subjected to SDS-PAGE. A gel piece was cut at the expected size without staining of the gel, alkylated with iodoacetamide, trypsin-digested and subjected to LC-MS/MS analysis.

Mass spectrometry analysis. LC-MS/MS was carried out by nanoflow reversed-phase liquid chromatography (RPLC) (Agilent) coupled online to a linear ion trap (LTQ)-Orbitrap XL mass spectrometer (Thermo). Briefly, the LC separation was performed using a PicoFrit analytical column $(75 \mu \mathrm{m} \mathrm{ID} \times 150 \mathrm{~mm}$ long, $15-\mu \mathrm{m}$ Tip ID (New Objectives)) packed in-house with 3- $\mu \mathrm{m}$ C18 resin (Reprosil-AQ Pur). Peptides were eluted using a linear gradient from $2 \%$ to $40 \% \mathrm{~B}$ over $60 \mathrm{~min}$ at a flow rate of $200 \mathrm{nl} / \mathrm{min}$ (solvent A: $97.9 \% \mathrm{H}_{2} \mathrm{O}, 2 \%$ acetonitrile $0.1 \%$ formic acid; solvent B: $97.9 \%$ acetonitrile, $2 \% \mathrm{H}_{2} \mathrm{O}, 0.1 \%$ formic acid). $1.8 \mathrm{kV}$ was applied for nanoelectrospray generation. CID fragmentation was used when acquiring MS/MS spectra consisting of an Orbitrap full MS scan $(300-2,000 \mathrm{~m} / z$, resolution of 60,000 at $\mathrm{m} / z 400$ ) followed by up to 10 LTQ MS/MS spectra (TOP10) in the linear ion trap with normalized collision energy (setting of 35\%) on the most abundant ions detected in the full MS scan. Target ions already selected for MS/MS were dynamically excluded for $10 \mathrm{~s}$.

18. Goehler, H. et al. Mol. Cell 15, 853-865 (2004).

19. David, M., Dzamba, M., Lister, D., Ilie, L. \& Brudno, M. Bioinformatics 27, 1011-1012 (2011).

20. Stelzl, U. et al. Cell 122, 957-968 (2005).

21. Huang, da W., Sherman, B.T. \& Lempicki, R.A. Nat. Protoc. 4, 44-57 (2009).

22. Palidwor, G.A. et al. PLoS Comput. Biol. 5, e1000304 (2009).

23. Passos, D.0., Quaresma, A.J. \& Kobarg, J. Biochem. Biophys. Res. Commun. 346, 517-525 (2006).

24. Lee, J. \& Bedford, M.T. EMBO Rep. 3, 268-273 (2002).

25. Lee, J., Cheng, D. \& Bedford, M.T. Methods Mol. Biol. 284, 195-208 (2004). 